\title{
Inner speech, secondary planner in Tower of London task in natural old age
}

\author{
Maryam Atabati ${ }^{1}$, Nader Jahangiri ${ }^{2}$, Naghmeh Mokhber $^{3}$ \\ ${ }^{1}$ Department of Linguistics, Ferdowsi University of Mashhad, Mashhad, Iran \\ ${ }^{2}$ Ferdowsi University of Mashhad, Mashhad, Iran \\ ${ }^{3}$ Psychiatry and Behavioral Sciences Research Center of Mashhad University of Medical Sciences, Mashhad, Iran \\ Email: Maryam.atabati@yahoo.com
}

Received 23 August 2011; revised 24 October 2011; accepted 7 November 2011

\begin{abstract}
In this study the mechanical version of the three-disk Tower of London task with changes in the movements was conducted by fifteen elderly participants with concurrent articulatory suppression. Also, this executive task was conducted without verbal secondary task and the results of these two states were compared with each other. From this comparison, got evidences based on inner speech role in more complicated Tower of London tasks, although in general, the results suggest a more outstanding role of inner scribe in spatial planning in this executive task. Then inner speech and inner scribe roles have been described in Tower of London task applying "Baddeley and Logie" working memory model.
\end{abstract}

Keywords: Inner Speech; Tower of London Task; Phonological Loop; Visuospatial Sketchpad; Articulatory Suppression

\section{INTRODUCTION}

We are required to handle information in our working memory for thinking and solving problems. In the model of working memory proposed by Baddeley and his colleagues [1], the central executive coordinates two storage systems, visuospatial sketchpad and phonological loop. Spatial working memory has two subcomponents; a spatial rehearsal and visual cache, and verbal memory is fractionated to verbal rehearsal and phonological store. According to Vygotsky [2], inner speech has a specific role in planning and facilitates problem solving. The executive Tower of London (TOL) task is used for evaluating of planning in order to establish whether visuospatial sketchpad or phonological loop could be evidenced with functioning of rehearsal and storage. A solver can compare the goal state with the start and a solution can resemble these two states. Saying repeatedly some words or phrase or articulatory suppression overloads phonological loop and prevents from mental rehearsing [3]. Some researchers suggested the importance of full mental preplanning in TOL trials [4,5]. Ward and Allport [5] found the relation between preparation time and performance [6]. If articulatory suppression impairs performance on TOL, inner speech will show it's affirmative role in executive task. In Wallace and co-worker's experiment [7], with a group of typically developing adolescents (12- to 19-year-old), articulatory suppression was resulted in more moves to reach solutions and detrimentally affected TOL performance but in adolescents with autism disorders, articulatory suppression did not significantly diminish performance. These findings supported this idea that children with autism who have executive dysfunction, maybe have impaired inner speech too.

Phillips and colleagues [8] emphasized on concurrent planning of TOL trials. In their views, preplanning might result in verbally rehearsed plans and inner speech did not show positive role in their experiments. With controlling dual task in young adults (18- to 25-year-old), completing five-disk Tower of London task [9], they suggested that articulatory suppression reduced preplanning times and increased speeds of performance. Also, it was not detrimental to number of excess moves. During two Lidstone's experiment [10] in Children (7- to 10year-old) on three-disk Tower of London task [11] was shown that under articulatory suppression, performance was lower than control condition, just when solvers were forced to plan a head but not in normal condition. Authors believed that easy beginning (two and three moves apparatus) was likely one reason for little planning in normal condition. Another component of working memory, visuospatial sketchpad holds visual and spatial information separately [12]. For spatial planning, spatial rehearsal should be done. Therefore, spatial information should be kept available. In other words, active maintenance is necessary for problem solving. Awh and Jonides 
[13] argued that covert shifts of attention play a role in spatial rehearsal, they considered the potential role of implicit motor programs to serve as an engram for spatial memory, a functional overlap in the mechanisms of spatial working memory and spatial selective attention. However, evidence for motor-based and attention-based rehearsals are different tasks and motor based rehearsal has been occurred with task that the positions are recalled via directed motor movements [13]. Smyth and Scholey [14] suggested that maintenance in visuospatial immediate memory is based on shifts of spatial attention rather than the operation of implicit motor processes. Godijn and Theeuwes [15] with conducting two tasks to memorize the locations of a number of digits argued that overt eye movements did not any benefit over covert attention for rehearsaling visuospatial information. According to Baddeley and colleagues [3] implicit eye movement programs mediates locations in spatial working memory as covert articulation mediates rehearsal in verbal memory. Osaka and colleagues suggested that [16] temporary retention of sequential locations and movements by an active spatial rehearsal mechanism, "inner scribe" in the model of working memory proposed by Logie [17] has overlap with process underlying the planning without overt movement and production of physical movement. One way for investigating the role of subvocal rehearsal on executive tasks, is showing, how second verbal task can influence spatial planning. For this reason, we should increase difficulties of the task gradually. In this way, we follow up the optimum strategy of working memory through articulatory suppression and blocking of inner speech. Therefore, that is possible, inner speech comes up in situations for planning, which is linked to points that we try to respond them in this examination.

\section{METHOD}

\subsection{Participants}

A total of 15 elderly participants aged $62-70$ years (11 men and 4 women, mean of age $=64 / 93$ and s.d. $=2 / 218$ ) were selected for this trial from the different parts of the society of Mashhad. They were healthy with apparently normal cognition and the MMSE score for them was higher than 26.

\subsection{Procedure}

Participants were seated on the bench with the examiner. Two identical apparatus were in the middle part of the bench with the short distance to each other. Film taking fom the whole procedure of the trial was done by the small camera. They were told about the practice and main trial for recording. For anybody, the examiner explained about the methods and rules of the trial. Then participants tried to solve all of the 11 different configurations that they were presented one by one. Some of the participants dissolved apparatus from standard to target and some of them dissolved reversely from target to standard. Training of the participants was done in two forms. Time of doing trials measured with the accuracy of second hundredth. Measurement of time was started from picking up the first bead.

\subsection{Task}

Because using of this test for the elderly, other innovative movements was necessary so that we could increase measurement possibility of time and motion correctness. Thus, with the extension of possible movements minimum for solving the apparatus, we attained to 6-moves and 7-moves apparatus which they can be solved in a minimum of six and seven moves. In general we used different configurations from the usual twelve configurations and 2- moves and 3-moves apparatus were simple and most of the participants could solve them quickly, but the new configurations were suitable for the elderly. These configurations were characterized with insertion of movement number 4, 5, 6, 7 beside the capital letters A-B-C-D-E. Therefore we used from 11 different configurations including (4A, B moves), that is, two kinds of 4-moves and (5A, B, C, D, E moves), five kinds of 5moves and one kind of 6-moves and (7A, B, C), three kinds of 7-moves. These figurations were propounded on three-disk Tower of London task [11]. Participants were not stopped with the first wrong movements and they were just stopped with the role breaking like concurrent moving of two balls or with the dropping of the balls. Two identical copies of Tower of London task were made, according to dimensions mentioned in the research of Fernyhough and colleagues [18]. Each apparatus consisting of three metal rods of different lengths, mounted on a wooden base $(20 \mathrm{~cm} \times 7 \mathrm{~cm} \times 2 \mathrm{~cm})$, three coloured beads (red, green and blue). The lengths of the rods were such that the highest could be occupied by three beads and the central by two beads and the smallest by one bead. The standard apparatus was always fixed. The highest rod accommodates two beads, upper red and below green and central rod accommodates one blue bead. The target apparatus was set up in one of the 11 different configurations every time.

\section{TOWER OF LONDON TASK (TOL)}

Some researchers applied dual task methodology to explore the role of working memory on the TOL. Morris and colleagues [19] argued that verbal working memory is loaded for planning in the TOL because it involves left frontal lobe but Phillips and co-workers [8] suggested that visuospatial components and central executive are 
involved in TOL performance in young participants. According to Phillips and colleagues [20] the conditions of older participants were different and performing the TOL loaded all components of working memory. Articulatory suppression loads working memory phonological loop [3]. This secondary task caused poorer TOL performance in old compared to young participants. Gilhooly and co-workers [21], using think-aloud methods cited older participants were less able to plan a head on the TOL than younger participants, but there was no difference between them in the moving phase. Gilhooly and colleagues [22] did not find any evidence of verbal working memory involvement in the TOL. They explained that the left frontal lobes were involved in visuospatial memory, but the predominant strategy in the TOL was executed using spatial code and was held by the active spatial rehearsal mechanism, inner scribe. Because the TOL task measures in the correlation matrix was correlated most with Corsi and Distance task measures that engage the active spatial subcomponent of visuospatial working memory. In this experiment, we apply one secondary verbal task in combination with the TOL, and then results are compared with the single TOL examination by the same participants. In this way, the involvement of the phonological loop and inner speech can be revealed on the TOL performance.

\section{RESULT AND DISCUSSION}

In this experiment, participants should change apparatus in minimum number of moves and time without rulebreaking. Thus, a pair measures were used for each trial: moves and time to solution. Some of them were instructed with articulatory suppression to start the practice and the others without that. Those participants that started with articulatory suppression were predominated soon. The results were inserted to tables in terms of excess moves and time. Therefore the best result for the excess moves column was zero and the best time was the least time. Participants were not stopped with the wrong moves that they were becoming far from the goal and all of the moves were recorded. For example, it was possible that 7-moves apparatus be solved in 10 moves. Participants were not successful to dissolve all of the configurations. Several cases of rule breaking, dropping the beads, putting beads beside the rod were seen that did not record any result for them. All of the configurations were presented to the participants, and each solver was able to dissolve some of them. Participants dissolved the TOL task in a single task condition and in repeatedly saying three words in time. Then the mean of two conditions were compared with two excess moves and time column diagrams. The dual task results determined with insertion of 1 and the single task results determined with insertion of 2. The means of excess moves and time were attained in two steps. First, amongst the each of 4, 5, 6, 7-moves trials (Tables 1-4) and second, amongst the whole of them in the single and dual task separately (Table 5). In the single trials, participants carried out silently or with self-directed speech. It was possible that participants explain their planning and the manner of doing trials for themselves. Thus, solver could plan mentally from starting time of the moves. We want to know about the efficiency of solvers through comparison of accuracy and speed of doing trials in single and dual task condition. Whatever participants have dissolved apparatus in fewer time and fewer movements the time and excess column are lower, and they indicate more value of solver speed and ability.

In 4-moves configurations, the spending time to dissolve with articulatory suppression was fewer than the conditions with verbal freedom (Figure 1). That is, participants with prohibition of inner speech have carried out 4-moves trials faster. Excess movement numbers of

Table 1. Time and excess moves of 4-moves apparatus in dual (4.1) and single (4.2) task.

\begin{tabular}{ccc}
\hline mean & 4.1 & 4.2 \\
time & 7.96 & 11.32 \\
Excess moves number & 0.182 & 0.583 \\
\hline
\end{tabular}

Table 2. Time and excess moves of 5-moves apparatus in dual (5.1) and single (5.2) task.

\begin{tabular}{ccc}
\hline mean & 5.1 & 5.2 \\
time & 14.35 & 17.92 \\
Excess moves number & 1.19 & 2.233 \\
\hline
\end{tabular}

Table 3. Time and excess moves of 6- moves apparatus in dual (6.1) and single (6.2) task.

\begin{tabular}{ccc}
\hline mean & 6.1 & 6.2 \\
time & 15.791 & 36.853 \\
Excess moves number & 0.50 & 5.62 \\
\hline
\end{tabular}

Table 4. Time and excess moves of 7-moves apparatus in dual (7.1) and single (7.2) task.

\begin{tabular}{ccc}
\hline mean & 7.1 & 7.2 \\
time & 23.008 & 23.477 \\
Excess moves number & 1.7079 & 1.5778 \\
\hline
\end{tabular}

Table 5. Time and excess moves of all apparatus in dual (1) and single (2) task.

\begin{tabular}{ccc}
\hline mean & 1 & 2 \\
time & 15.28 & 22.39 \\
Excess moves number & 0.895 & 2.505 \\
\hline
\end{tabular}


participants for dissolving 4-moves apparatus with articulatory suppression was fewer than single task approach (Figure 2). In other word, negative effect of inner speech on performance of solvers is obvious.

Dissolving time of 5-moves apparatus with articulatory suppression has been fewer than single task conditions (Figure 3). That is, positive effect of articulatory suppression on rapidity of solver performance is obvious.
Also, participants have had fewer excess moves number in solving of 5-moves apparatus with articulatory suppression (Figure 4). That is, participants have dissolved these apparatus with stopping inner speech from the more convenient way. They have done fewer movements that they become far from the main goal.

Maximum of spending time for 6-moves apparatus dissolving has related to single task condition (Figure 5).

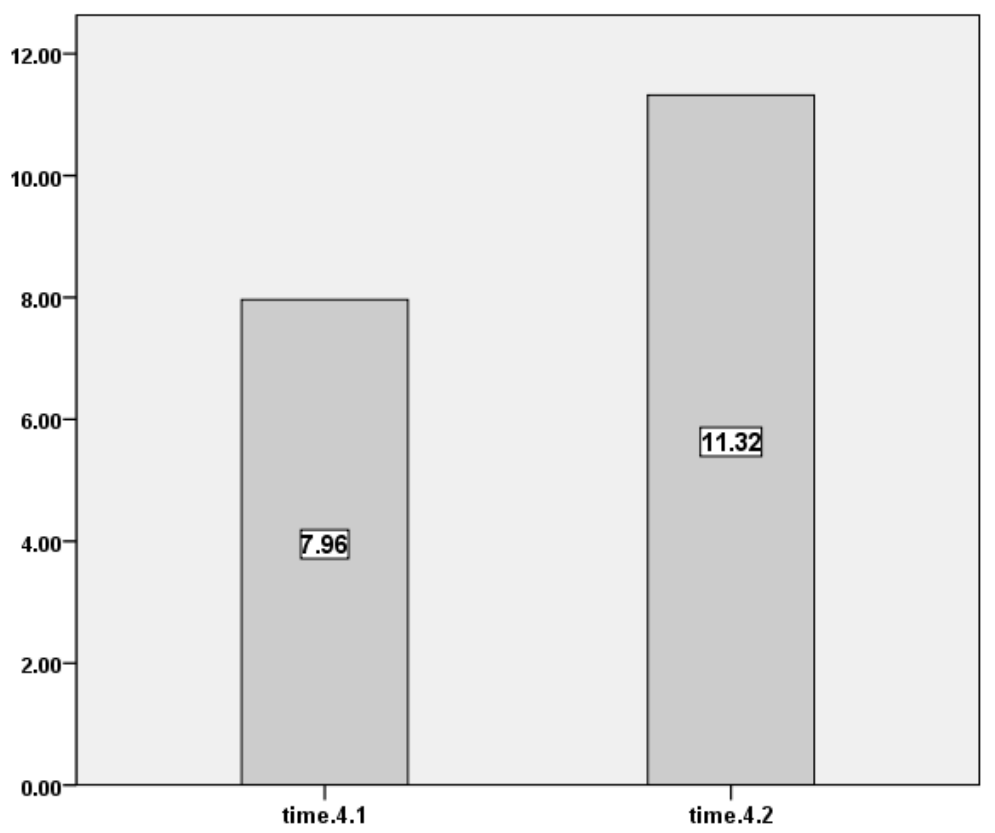

Figure 1. The comparison of spending time for dissolving of 4-moves apparatus in dual (1) and single (2) task condition.

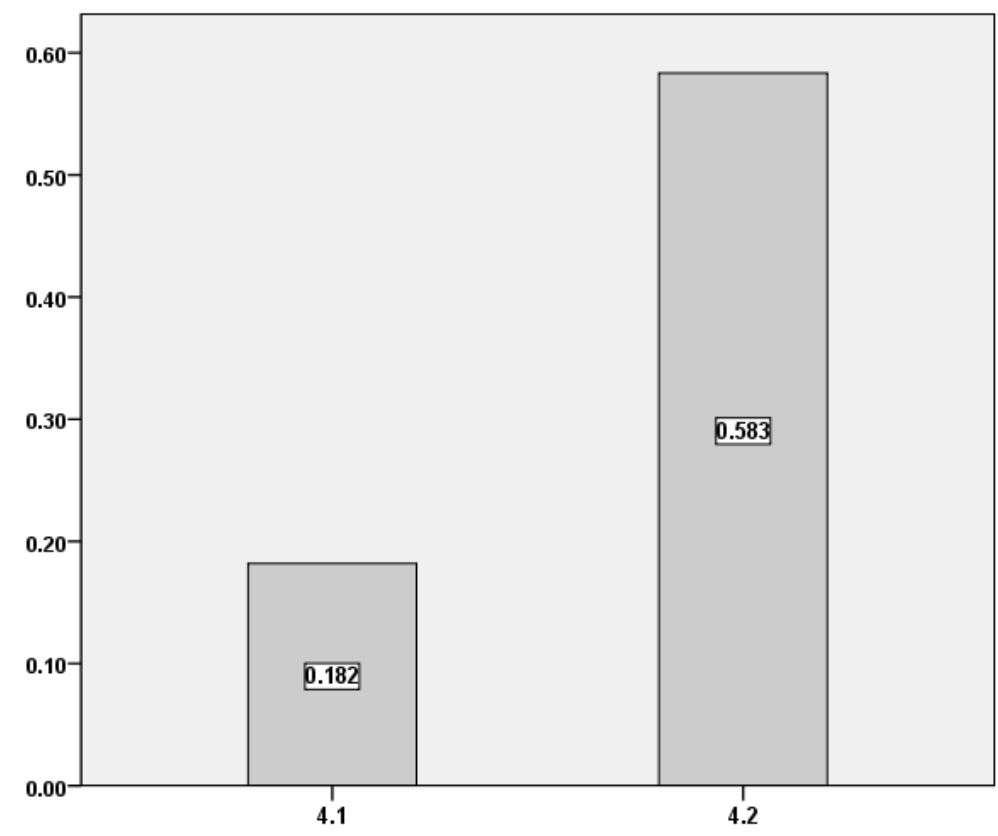

Figure 2. The comparison of excess moves numbers for dissolving of 4-moves apparatus in dual (1) and single (2) task condition. 


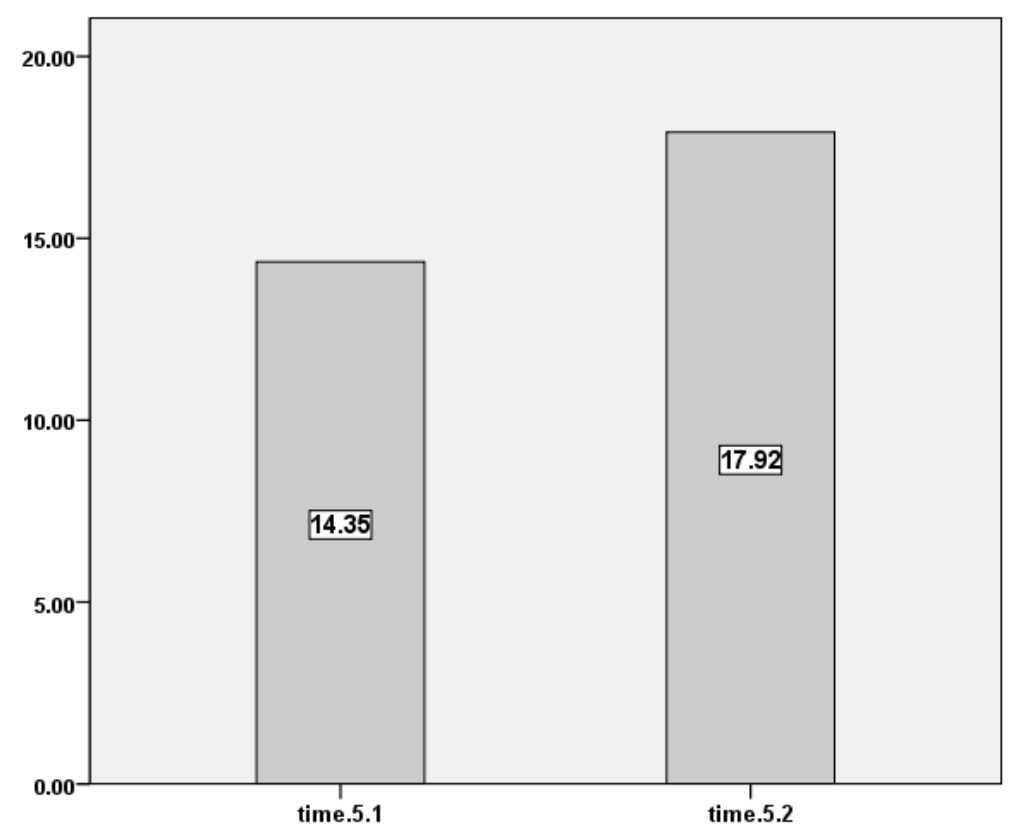

Figure 3. The comparison of spending time for dissolving of 5-moves apparatus in dual (1) and single (2) task condition.

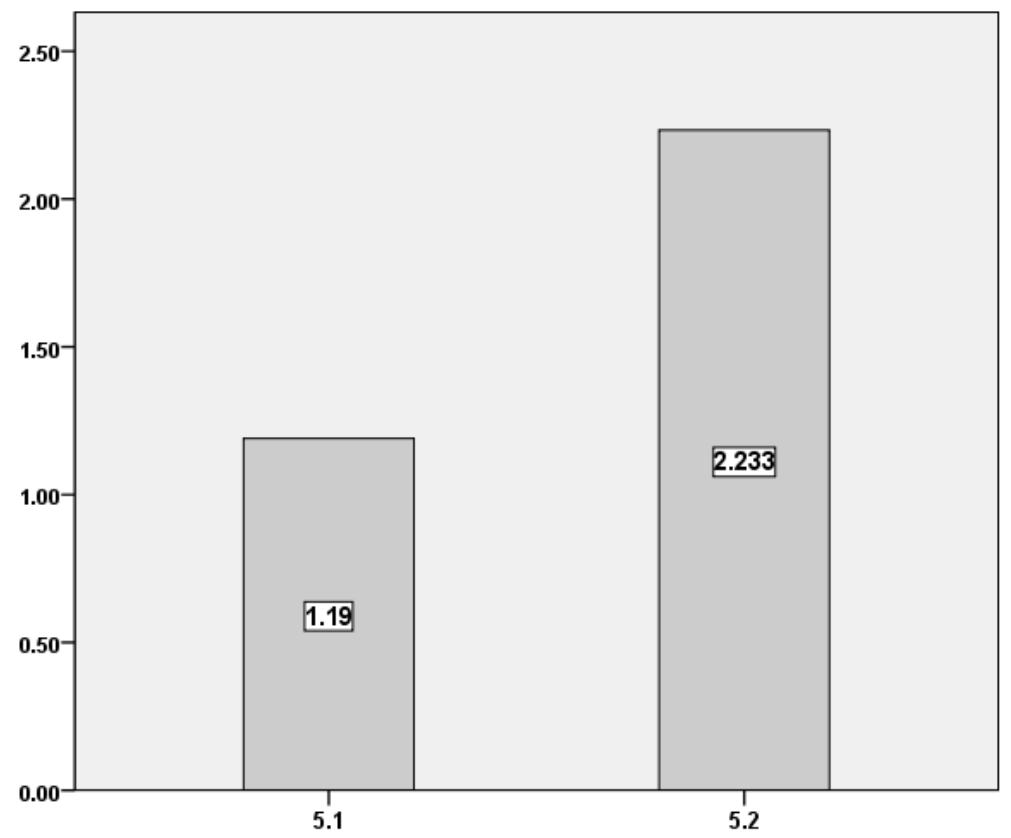

Figure 4. The comparison of excess moves numbers for dissolving of 5-moves apparatus in dual (1) and single (2) task condition.

Participants have had more rapidity of performance and fewer excess movements with articulatory suppression (Figure 6).

With becoming more complicated of movements about 7-moves apparatus, time of two states are becoming equal. In other words, dissolving time of 7-moves apparatus in single task conditions are approximately the same as dissolving time of dual task conditions (Figure
7), and excess moves of dual task conditions is more than the single task conditions (Figure 8). That is, articulatory suppression has not had positive effect on solvers of 7 moves apparatus. In additional, for dissolving of 7-moves apparatus, we can express explicitly positive effect of inner speech for planning ahead. It can be mentioned that in dissolving of more difficult apparatus, inner speech shows it's function. 


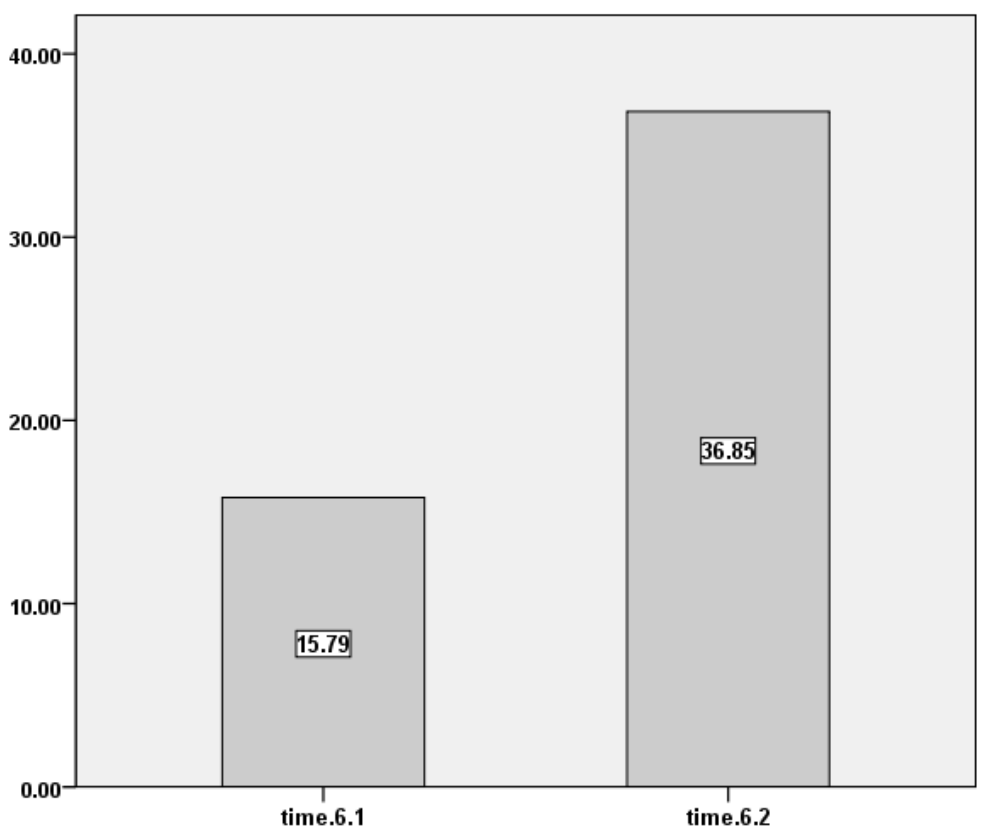

Figure 5. The comparison of spending time for dissolving of 6-moves apparatus in dual (1) and single (2) task condition.

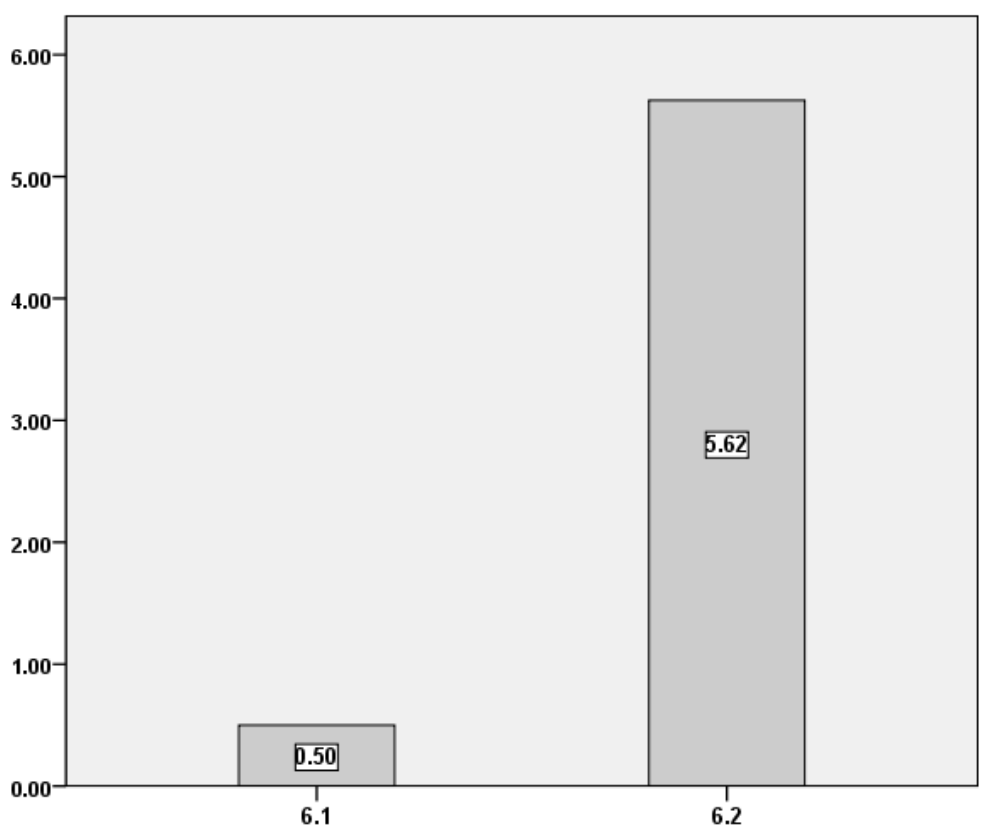

Figure 6. The comparison of excess moves numbers for dissolving of 6-moves apparatus in dual (1) and single (2) task condition.

Above tables (Tables 6 and 7) show the amount of paired samples test for two related groups and $\alpha=0.00$, then difference of spending time and excess moves numbers in dual (1) and single (2) task is significant.

In the case of general performance of solvers, the positive effect of articulatory suppression on both numbers of excess moves (Figure 10) and spending time (Figure 9) is obvious.
Table 6. Paired samples test (time).

\begin{tabular}{|c|c|c|c|c|c|}
\hline \multicolumn{6}{|c|}{ Paired Samples Test } \\
\hline & \multicolumn{2}{|c|}{ Paired Differences } & \multirow{2}{*}{$\mathrm{t}$} & \multirow{2}{*}{ df } & \multirow{2}{*}{ Sig. (2-tailed) } \\
\hline & Mean & Std. Deviation & & & \\
\hline time 1 - time 2 & -7.11 & 8.67717 & -3.173 & 14 & 0.00 \\
\hline
\end{tabular}




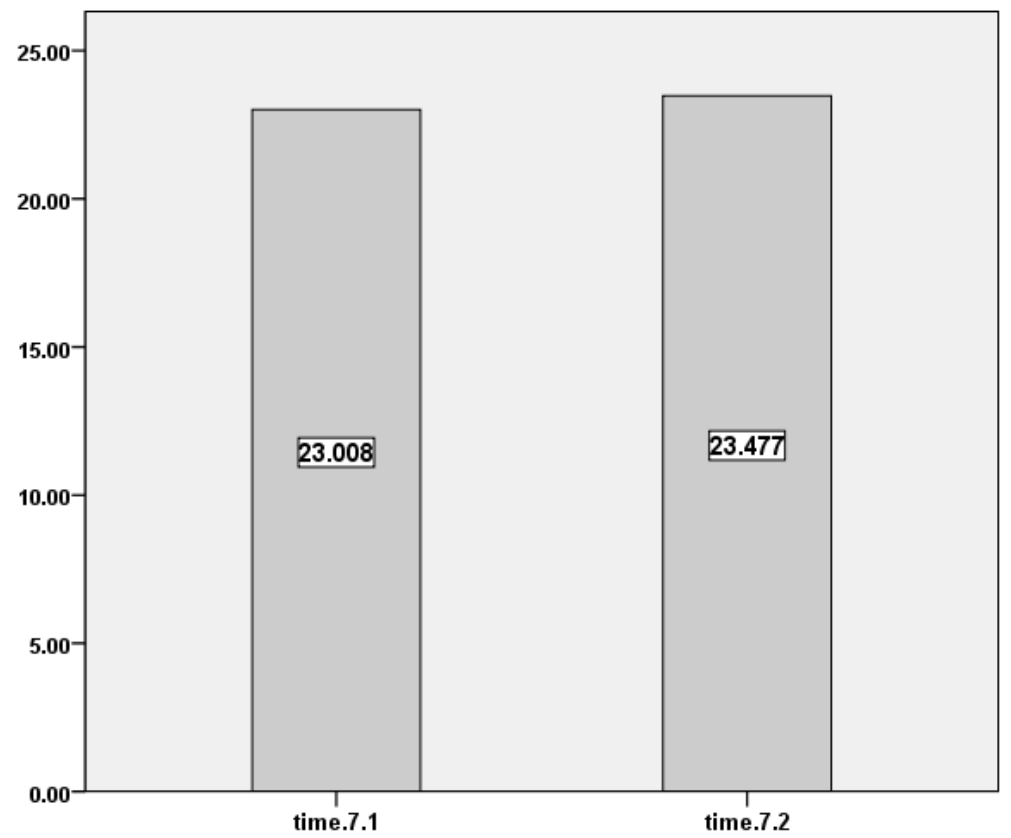

Figure 7. The comparison of spending time for dissolving of 7-moves apparatus in dual (1) and single (2) task condition.

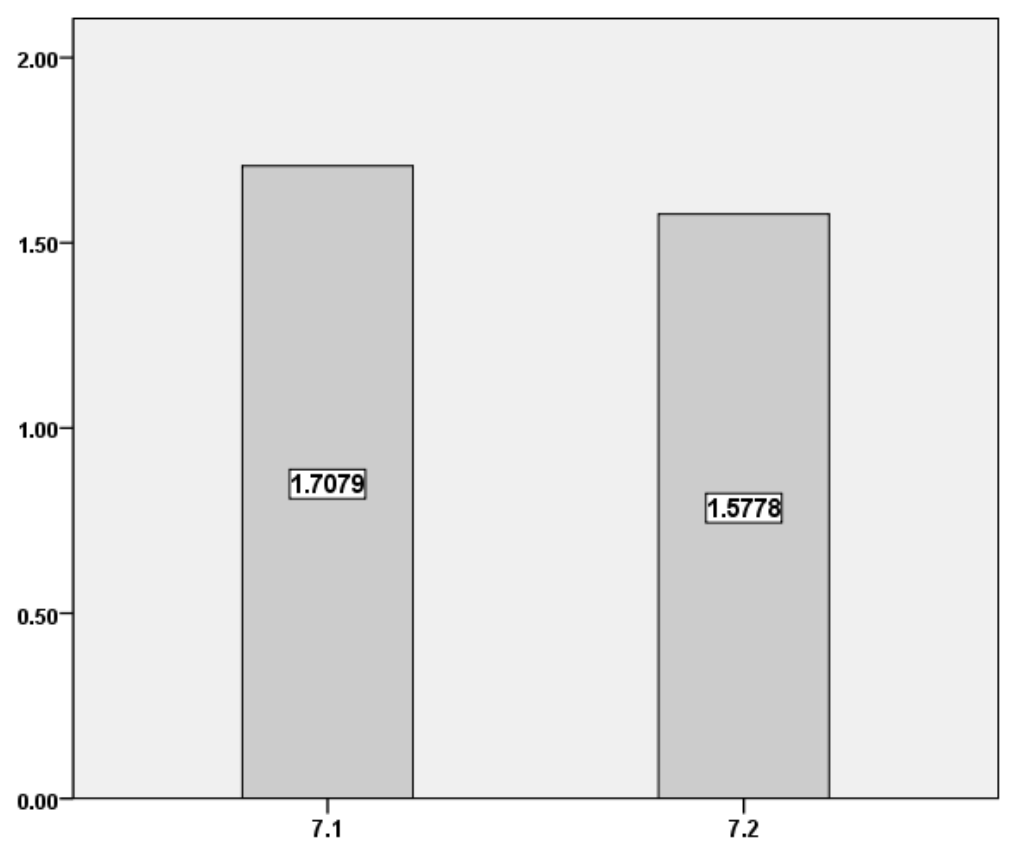

Figure 8. The comparison of excess moves numbers for dissolving of 7-moves apparatus in dual (1) and single (2) task condition.

Table 7. Paired samples test (move).

\begin{tabular}{|c|c|c|c|c|}
\hline \multicolumn{5}{|c|}{ Paired Samples Test } \\
\hline & \multicolumn{2}{|c|}{ Paired Differences } & \multirow{2}{*}{$\mathrm{t} \quad \mathrm{df}$} & \multirow{2}{*}{ Sig. (2-tailed) } \\
\hline & Mean & Std. Deviation & & \\
\hline move 1 - move 2 & -1.61 & 1.299 & $\begin{array}{ll}-4.8 & 14\end{array}$ & 0.00 \\
\hline
\end{tabular}

\section{DISCUSSION}

Participants move beads one by one to convert the start state to the goal state, the start state was standard or 4, 5, 6, 7-moves configuration.

That is, some solvers started from variable state and then they tried to reach to a fixed state. About these participants, the effect of inner speech suppression on the 


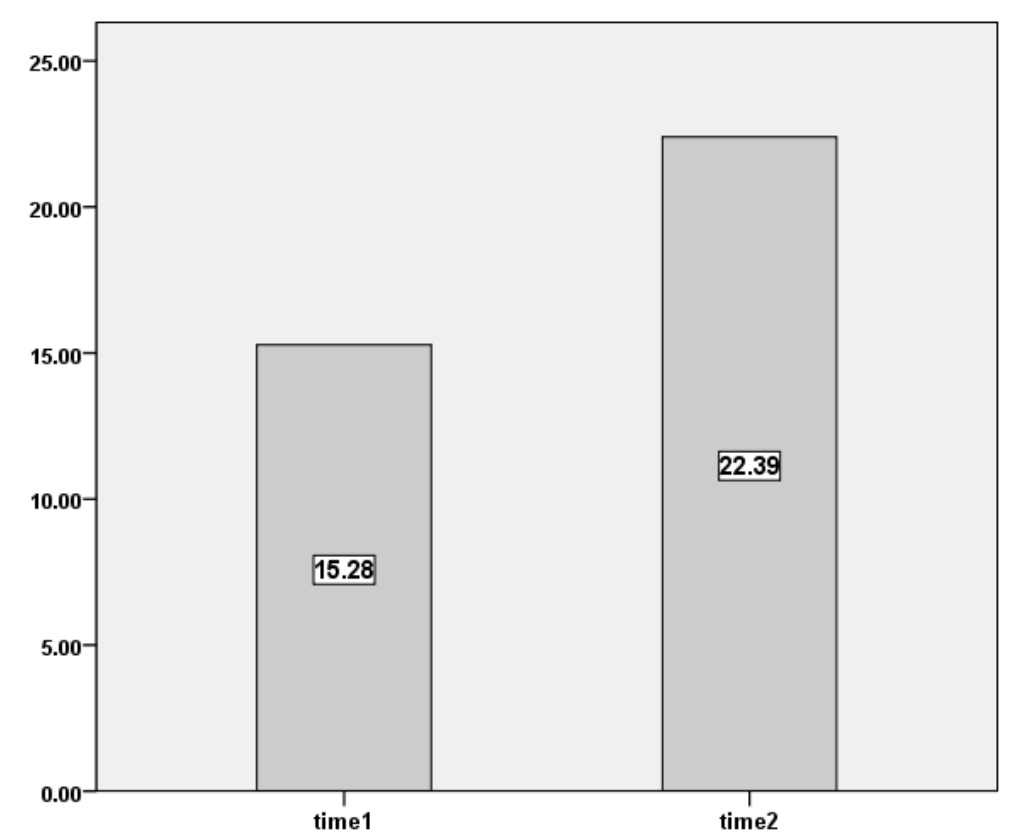

Figure 9. The comparison of spending time for dissolving of all apparatus in dual (1) and single (2) task condition.

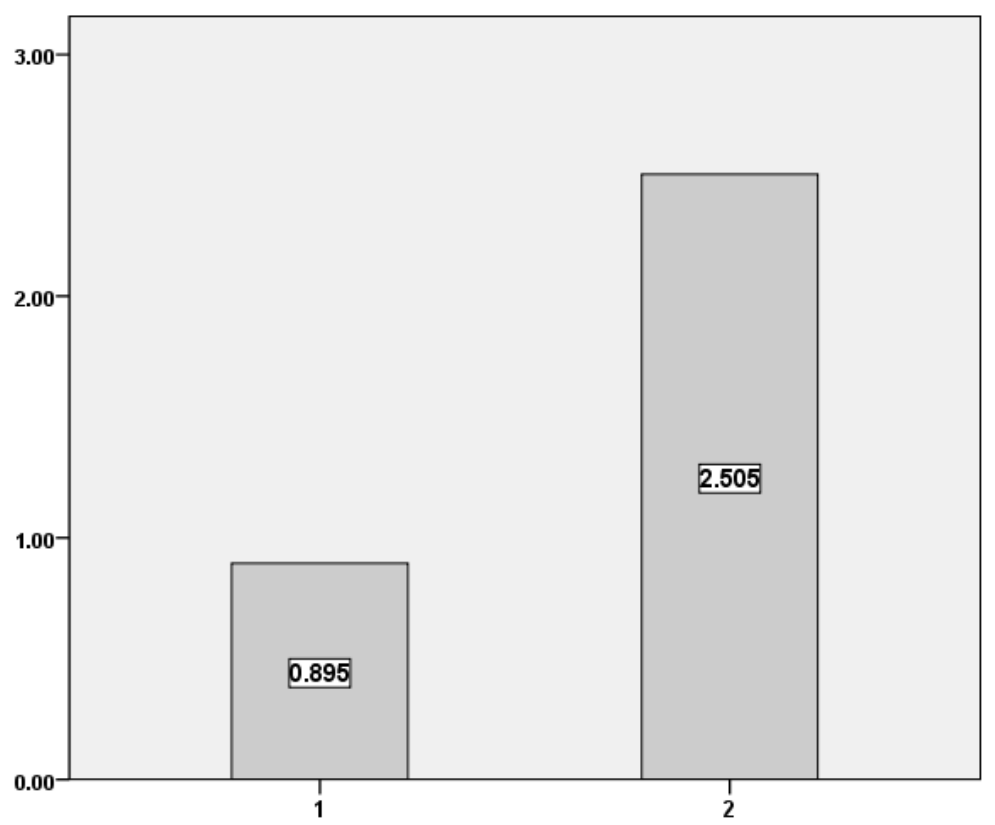

Figure 10. The comparison of excess moves numbers for dissolving of all apparatus in dual (1) and single (2) task condition.

results was more obvious than the other participants who started from standard state. When participants started from variable configurations, their performance with articulatory suppression was higher than participants who started from a fixed state. We changed the movements of three-disk Tower of London task [11] and more complicated configurations were used. Therefore articulatory suppression didn't always improve performance, and some times solvers were confused. Since, articulatory suppression negates possibility of verbal preplanning, solver start to move the beads sooner. Phillips and colleagues [20] argued that making verbally based plans on the TOL, is ineffective because reduced planning time under articulatory suppression did not impair the accuracy of TOL performance. They suggested that prevent from verbal rehearsing during planning, encourage the 
use of an optimal spatial strategy involving the visuospatial scratchpad [8]. We don't argue about the children's inner speech that it seem have role on even easier apparatus dissolving.

\section{CONCLUSIONS}

According to the multiple-component model of working memory $[23,24]$, the central executive can process activated perior knowledge representations with the single representation from both of the vebal and visuospatial working memory. In Tower of London task, information should be combined in this manner. When silent verbal is suppressed through articulatory suppression, namely inner speech is omitted from working memory processing cycle. It is possible that just visual information from the visual cache enter the central executive for processing. This matter is related to attend or ignore the stopping stimulus by working memory central executive of person. Repeatedly saying a phrase or some word sequence, although prevents from articulatory rehearsal, it is a kind of input that loads phonological loop instead of silent verbal. That is, this input enters the phonological store instead of inner speech. This is related to central executive that to what extent can ignore the stopping stimulus. If central executive selectively attend to stopping stimulus, part of it's performance will be spent for processing of stopping stimulus, but if central executive can ignore the stopping stimulus, just visual information from the visual cache with the activated prior knowledge are processed. For example, saying the digits 1 to 9 repeatedly is easy task, but random number generation is second hard task. About 4, 5, 6-moves apparatus, execution output of task with articulatory suppression is more than the output of single task condition. That is, about simpler apparatus, not only, inner speech has not had any positive effect, but also prohibition of its articulation has provided more appropriate way and further speed for elderly participants. However, bead moving time in 7moves apparatus has become approximately equal in single and dual task condition. That is, articulatory suppression has not given further speed to solvers. Especially in the field of number of excess moves made, we can tell that articulatory suppression has had negative effect on participants. Here, the role of inner speech is specified on executive tower task. Thus, when inner speech play the role of planning ahead in TOL task better, which named tasks be more difficult, but we should consider an extent for difficulties, because participants might be frustrated about dissolving and desist. In Tower of London task, the role of inner speech is verbal rehearsal and the role of inner scribe is spatial rehearsal of the task. So, because inner scribe is active spatial rehearsal system, can maintain sequential situations and movements. It can rehearse motion lines in Tower of London task and follow them until the goal. That is, in elimination of verbal rehearsal system, spatial rehearsal system is responsible outstandingly for visual signs retrieval of nonverbal memory.

\section{REFERENCES}

[1] Baddeley, A. and Sala, S.D. (1996) Working memory and executive control. Philosophical Transactions of the Royal Society of London, Series B, Biological Sciences, 351, 1397-1403.

[2] Vygotsky, L.S. (1962) Thought and language. The MIT Press, Cambridge. doi:10.1037/11193-000

[3] Baddeley, A.D. (1986) Working Memory. Oxford University Press, Oxford.

[4] Owen, A.M. (1997) Cognitive planning in humans: Neuropsychological, neuroanatomical and neuropharmacological perspectives. Progress in Neurobiology, 53, 431-450.

[5] Ward, G. and Allport, A. (1997) Planning and problem-solving using the five disc Tower of London task. Quarterly Journal of Experimental Psychology Section A: Human, 50, 49-78. doi:10.1080/713755681

[6] Morris, R. and Ward, G. (2005) The cognitive psychology of planning. Psychology Press, Hove.

[7] Wallace, G.L., Silvers, J.A., Martin, A. and Kenworthy, L.E. (2009) Brief report: Further evidence for inner speech deficits in autism spectrum disorders. Journal of Autism and Developmental Disorders, 39, 1735-1939.

[8] Phillips, L.H., Wynn, V., Gilhooly, K.J., Sala, D.S. and Logie, R.H. (1999) The role of memory in the tower of london task. Memory, 7, 209-231.

[9] Ward, G. and Allport, A. (1997) Planning and problem-solving using the five disc Tower of London task. Quarterly Journal of Experimental Psychology Section A: Human, 50, 49-78. doi:10.1080/713755681

[10] Lidstone, J.S.M., Meins, E. and Fernyhough, C. (2010) The roles of private speech and inner speech in planning during middle childhood: Evidence from a dual task paradigm. Journal of Experimental Child Psychology, 107, 438-451. doi:10.1016/j.jecp.2010.06.002

[11] Shallice, T. (1982) Specific impairment of planning. Philosophical Transactions of the Royal Society of London. Series B, Biological Sciences, 298, 199-209. doi:10.1098/rstb.1982.0082

[12] Tresch, M.C., Sinnamon, H.M. and Seamon, J.G. (1993) Double dissociation of spatial and object visual memory: Evidence from selective interference in intact human subjects. Neuropsychologia, 3, 211-219. doi:10.1016/0028-3932(93)90085-E

[13] Awh, E. and Jonides, J. (2001) Overlapping mechanisms of attention and spatial working memory. Trends in Cognitive Sciences, 5, 119-126. doi:10.1016/S1364-6613(00)01593-X

[14] Smyth, M.M. and Scholey, K.A. (1994) Interference in immediate spatial memory. Memory \& Cognition, 22, 1-13. doi:10.3758/BF03202756 
[15] Godijn, R. and Theeuwes, J. (2012) Overt is no better than covert when rehearsing visuo-spatial information in working memory. Memory \& Cognition, 40, 52-61.

[16] Osaka, N., Logie, R.H. and Esposito, M.D. (2007) The cognitive neuroscience of working memory. Oxford University Press, Oxford.

[17] Logie, R.H. (1995). Visuo-spatial working memory. Lawrence Erlbaum Associates Ltd., Hove.

[18] Fernyhough, C. and Fradley, E. (2005) Private speech on an executive task: Relations with task difficulty and task performance. Cognitive Development, 20, 103-120.

[19] Morris, R.G., Ahmed, S., Syed, G.M. and Toone, B.K. (1993) Neural correlates of planning ability: Frontal lobe activation during the Tower of London test. Neuropsychologia, 31, 1367-1378. doi:10.1016/0028-3932(93)90104-8

[20] Phillips, L.H., Gilhooly, K.J., Logie, R.H., Sala, S.D. and Wynn, V.E. (2003) Age, working memory, and the Tower of London task. European Journal of Cognitive Psychology, 15, 291-312. doi:10.1080/09541440244000148

[21] Gilhooly, K.J., Phillips, L.H., Wynn, V., Logie, R.H. and Sala, S.D. (1999) Planning processes and age in the five disc Tower of London task. Thinking \& Reasoning, 5, 339-361. doi:10.1080/135467899393977

[22] Gilhooly, K.J., Wynn,V., Phillips, L.H., Logie, R.H. and Sala, S.D. (2002) Visuo-spatial and verbal working memory in the five-disc Tower of london task: An individual differences approach. Thinking \& Reasoning, 8, 165-178.

[23] Baddeley, A.D. and Logie, R.H. (1999) Working memory: The multiple component model. In: Miyake, A. and Shah, P., Eds., Models of Working Memory, Cambridge University Press, Cambridge, 28-61.

[24] Baddeley, A.D. (2000) The episodic buffer: A new component of working memory? Trends in Cognitive Sciences, 4, 417-423. doi:10.1016/S1364-6613(00)01538-2 